\title{
Extraction of cordycepic acid from the fruiting body of Cordyceps militaris (L.)
}

\author{
Tuong Ha Do, Hoang Chinh NguYen * \\ Faculty of Applied Sciences, Ton Duc Thang University, Ho Chi Minh City, Vietnam
}

\begin{abstract}
Cordycepic acid from the fruiting body of Cordyceps militaris (L.) Link has various beneficial biological properties. However, the extraction of cordycepic acid from the fruiting body of $C$. militaris is difficult with minimal output. This study is aimed at optimizing the extraction conditions in order to maximize the cordycepic acid content. In the present study, cordycepic acid from the fruiting body of $C$. militaris was extracted using different solvents (methanol, ethanol, acetone, or distilled water). Among the solvents tested, ethanol was identified as the most efficient solvent for the extraction, resulting in the highest cordycepic acid content. Response surface methodology was subsequently employed to optimize the extraction conditions and establish a reliable mathematical model for prediction. A maximum cordycepic acid content of $40.23 \mathrm{mg} / \mathrm{g}$ was obtained at an ethanol concentration of $26.4 \%$, an extraction time of $69.8 \mathrm{~min}$, and a ratio of ethanol to sample of $23.4: 1 \mathrm{ml} / \mathrm{g}$. This study suggests that the obtained extraction conditions are the most effective for the extraction of cordycepic acid from the fruiting body of $C$. militaris.
\end{abstract}

Key words: cordycepic acid, extraction, optimization, response surface methodology

\section{Introduction}

Due to the doubts raised about the safety of synthetic drugs, there is an increasing interest in the use of natural products from herbal plants and medicinal fungi (Franco et al., 2018; Jin et al., 2018). These natural sources provide many natural bioactive compounds that possess various biological activities such as antioxidant, anti-inflammatory, anti-bacterial, and anticancer actions (Złotek et al., 2016; Jin et al., 2018). In addition, these natural bioactive compounds have no apparent side effects on human health (Bao et al., 2018; Wang et al., $2018 b)$. Therefore, there is an ongoing search for new bioactive agents from natural sources.

Cordyceps militaris (L.) Link is one of the most valued fungi in East Asian countries. It has been widely used in traditional Chinese medicine as a folk tonic and a supplementary for the treatment of various diseases (Das et al., 2010; Wang et al., 2018a). The fungus contains many kinds of bioactive compounds such as cordy- cepin, cordycepic acid, polysaccharides, ergosterol, and trace elements of various elements $(\mathrm{K}, \mathrm{Ca}, \mathrm{Mg}, \mathrm{P}$, and Fe) (Shih et al., 2007; Das et al., 2010; Tang et al., 2018). Among these constituents, cordycepic acid (Dmannitol), an isomer of quinic acid with a polyol structure, is one of the main pharmacological components in the fruiting body of $C$. militaris due to its numerous biological activities including antioxidant, antihepatic fibrosis, antilipid peroxidation, and antibacterial actions (Das et al., 2010; Deng et al., 2013). Consequently, cordycepic acid extracted from $C$. militaris is an important therapeutic agent for several industries, in particular, the nutraceutical and pharmaceutical industries (Xiao et al., 2009; Deng et al., 2013; Lin et al., 2016). However, studies on the methods of extraction of cordycepic acid from fruiting body of $C$. militaris are not numerous. Hence, there is an urgent need to develop an effective method for maximizing the extraction efficiency of cordycepic acid from $C$. militaris for industrial applications.

\footnotetext{
* Corresponding author: Faculty of Applied Sciences, Ton Duc Thang University, Ho Chi Minh City, Vietnam; e-mail: nguyenhoangchinh@tdtu.edu.vn; dotuongha@tdtu.edu.vn
} 
Extraction is the initial step to obtaining bioactive compounds from biomass materials. The main objective of the extraction process is to maximize the concentration of target compounds (Do et al., 2014). In response to this challenge, different methods have been studied for the extraction of bioactive compounds from biomass materials. Studies have demonstrated that the extraction yield is strongly affected by the extraction solvent (Do et al., 2014; Kuppusamy et al., 2015). To extract bioactive compounds, solvents with different polarities, including methanol, ethanol, acetone, and water, have been widely used. However, the efficiency of each solvent depends on the source materials and the chemical nature of the desired compounds, which have different solubilities in different solvents (Turkmen et al., 2006; Do et al., 2014; Zlotek et al., 2016). It is therefore difficult to suggest a suitable extraction solvent for extracting bioactive compounds from individual materials. For C. militaris, most researches have focused on the extraction of cordycepin and polysaccharides (Song et al., 2009; Wang et al., 2014; Soltani et al., 2017). However, no study has compared the effect of different solvents on the extraction of cordycepic acid and optimized the conditions for extracting this bioactive compound.

This study investigated the effect of different solvents (methanol, ethanol, acetone, and water) on the extraction of cordycepic acid from the fruiting body of $C$. militaris. Response surface methodology (RSM) was then used to optimize the extraction conditions (solvent concentration, extraction time, and ratio of solvent to sample) for obtaining the maximum cordycepic acid content.

\section{Materials and methods}

\section{Materials and reagents}

Fresh fruiting bodies of Cordycep militaris were provided by Rivendell Biotech Ltd. (Ho Chi Minh City, Vietnam). C. militaris was identified by using rDNA-ITS sequencing with a single pair of primers 18S-ITS1-5.8SITS2-28S rDNA synthesized by Nam Khoa BioTek (Ho Chi Minh City, Vietnam). DNA sequences were then compared to sequences deposited in the National Center of Biotechnology Information (NCBI) server (http:// www.ncbi.nlm.nih.gov) with Entrez and BLAST (basic local alignment search tool). The fruiting bodies were dried at $10^{\circ} \mathrm{C}$ and stored at $4^{\circ} \mathrm{C}$ for further experiments. Solvents and other reagents were of analytical grade and obtained from ECOH Chemical Co. Ltd. (Miaoli, Taiwan).

\section{Extraction of cordycepic acid with different solvents}

The fruiting bodies of $C$. militaris were ground using a blender (EUPA TSK-935BAP, Tsann Kuen Enterprise Co., Ltd., Taiwan) to produce powder. To investigate the effect of various extraction solvents (methanol, ethanol, or acetone) on the cordycepic acid content, the fruiting body powder ( $2 \mathrm{~g}$ ) was immersed in $20 \mathrm{ml}$ of each solvent at $30^{\circ} \mathrm{C}$ for $60 \mathrm{~min}$ with stirring. After the extraction, the sample was centrifuged at $10000 \mathrm{rpm}, 4^{\circ} \mathrm{C}$ for $5 \mathrm{~min}$. The supernatant was then collected to determine the cordycepic acid content using the method described by Xiao et al. (2009). The extraction of cordycepic acid using hot water $\left(100^{\circ} \mathrm{C}\right)$ was also conducted to compare the content obtained with that obtained by using organic solvents. All experiments were performed in triplicate.

\section{Optimization of cordycepic acid extraction using RSM}

A four-level and three-factorial central composite design was used to investigate the effect of extraction parameters (solvent concentration, extraction time, and ratio of solvent to sample) on the cordycepic acid content. Extractions with various solvent concentrations (26.493.6\%), extraction times (26.4-93.6 $\mathrm{min}$ ), and ratios of solvent to sample $(6.6: 1-23.4: 1 \mathrm{ml} / \mathrm{g})$ were performed in 100-ml Erlenmeyer flasks. The cordycepic acid content was determined from samples taken from the extraction mixtures. The relationship between the determined cordycepic acid content and extraction parameters was established using the following quadratic equation:

$$
\begin{aligned}
Y & =\beta_{0}+\beta_{1} X_{1}+\beta_{2} X_{2}+\beta_{3} X_{3}+\beta_{11} X_{1}^{2}+\beta_{22} X_{2}^{2}+\beta_{33} X_{3}^{2}+ \\
& +\beta_{12} X_{1} X_{2}+\beta_{13} X_{1} X_{3}+\beta_{23} X_{2} X_{3}
\end{aligned}
$$

where $Y$ is the cordycepic acid content $(\mathrm{mg} / \mathrm{g}) ; X_{1}$ is the solvent concentration (\%); $X_{2}$ is the extraction time (min); $X_{3}$ is the ratio of solvent to sample $(\mathrm{ml} / \mathrm{g}) ; \beta_{0}$ is the regression coefficient for the intercept term; $\beta_{1}, \beta_{2}$, and $\beta_{3}$ are linear parameters; $\beta_{12}, \beta_{13}$, and $\beta_{23}$ are interaction parameters; and $\beta_{11}, \beta_{22}$, and $\beta_{33}$ are quadratic parameters. The model parameters were determined using the least-squares method (Nguyen et al., 2017). A mathematical model was then established to determine the optimal extraction conditions for obtaining the 
maximal cordycepic acid content using a canonical method (Nguyen et al., 2017). Minitab 16 (Minitab Inc., State College, PA, USA) was used to establish the empirical model, carry out an analysis of variance (ANOVA), and determine the optimal extraction conditions.

\section{Determination of cordycepic acid content}

The content of cordycepic acid was determined using the method described by Xiao et al. (2009) with slight modifications. Briefly, the extract $(0.5 \mathrm{ml})$ was diluted in $5 \mathrm{ml}$ of distilled water. One $\mathrm{ml}$ of diluted sample was then oxidized with $1 \mathrm{ml}$ of periodate potassium solution at $53^{\circ} \mathrm{C}$ for $10 \mathrm{~min}$. Two $\mathrm{ml}$ of $0.1 \% \mathrm{~L}$-rhamnose was subsequently added to the reaction mixture to remove the residual periodate potassium. The reaction mixture was then mixed with $4 \mathrm{ml}$ of Nash reagent at $50^{\circ} \mathrm{C}$ for 15 min before being cooled to room temperature. The absorbance of the reaction mixture was subsequently measured at $412 \mathrm{~nm}$ using a spectrophotometer (UV-Vis Jasco V-730, Jasco, USA). Distilled water was used as the control. The standard curve for cordycepic acid (SigmaAldrich, USA) was established to calculate the cordycepic acid content in the extract. The standard curve equation was $y=0.0089 x-0.0108$, where $R^{2}=0.9961$.

\section{Statistical analysis}

The data presented in Figure 1 and Table 1 is the averages of the three replicates with standard deviation (SD). This data was analyzed by an analysis of variance (ANOVA) with Duncan's multiple range test at $P$ values $\leq 0.05$ using SAS software ver 8.2 (SAS Institute, Cary, NC, USA).

\section{Results and discussion}

\section{Effect of different solvents on the cordycepic acid content}

This study investigated the effects of distilled water and three organic solvents (methanol, ethanol, and acetone) on the extraction efficiency of cordycepic acid from the fruiting body of $C$. militaris. As shown in Figure 1 and Table 1 , the use of ethanol resulted in the highest cordycepic acid content $(12.32 \mathrm{mg} / \mathrm{g})$, followed by methanol $(10.33 \mathrm{mg} / \mathrm{g})$, distilled water $(9.97 \mathrm{mg} / \mathrm{g})$, and acetone $(4.0 \mathrm{mg} / \mathrm{g})$. This indicated that the extraction of cordycepic acid was favored by highly polar solvents. The high solubility of cordycepic acid in ethanol, me thanol, and distilled water enhanced the mass transfer

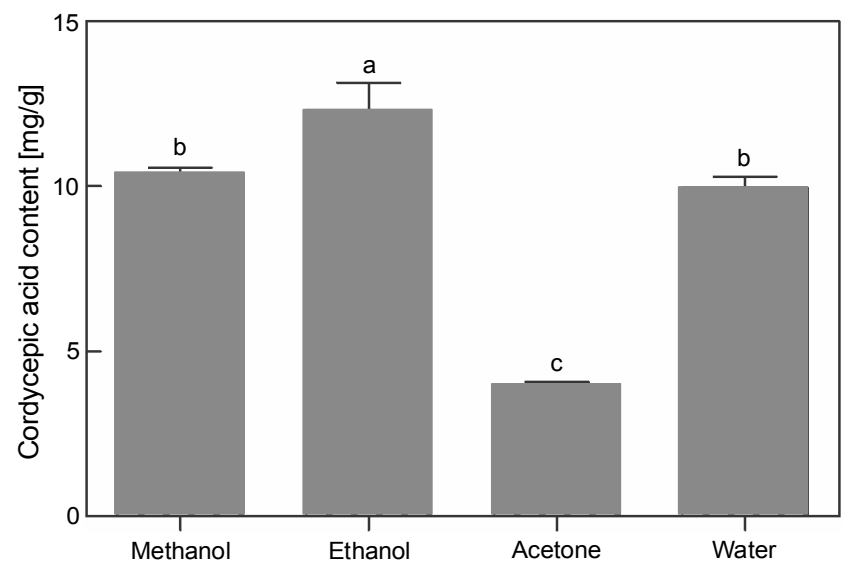

Fig. 1. Effects of different solvents on the cordycepic acid content in the extracts. The extractions were performed under the following conditions: $2 \mathrm{~g}$ of fruiting body powder, $20 \mathrm{ml}$ of solvent, and extraction time of $60 \mathrm{~min}$. The plotted values indicate the averages of three replicates with standard deviations. A, B, and C: different characters represent significant difference by Duncan's test at $P \leq 0.05$

Table 1. Effects of different solvents on cordycepic acid content in the extracts

\begin{tabular}{l|c}
\hline \multicolumn{1}{c|}{ Solvent } & $\begin{array}{c}\text { Cordycepic acid content } \\
{[\mathrm{mg} / \mathrm{g}]}\end{array}$ \\
\hline Methanol & $10.33 \pm 0.24^{\mathrm{b}}$ \\
\hline Ethanol & $12.32 \pm 0.82^{\mathrm{a}}$ \\
\hline Acetone & $4.00 \pm 0.07^{\mathrm{c}}$ \\
\hline Water & $9.97 \pm 0.32^{\mathrm{b}}$ \\
\hline
\end{tabular}

Data are expressed as the mean $\pm \mathrm{SD}$ of three replications; means within a column with different letters significantly differ by Duncan's test at $P<0.05$

that could occur during the extraction (Deng et al., 2013; Tahmouzi and Ghodsi, 2014). Another study has also shown that ethanol is an effective solvent for the extraction of cordycepic acid (Xiao et al., 2009). In addition, ethanol is considered to be eco-friendly, cheap, and non-toxic compared with methanol; therefore, it was selected for further experiments.

\section{Development of the RSM model}

In the present study, the relationship between cordycepic acid (measured response) and three extraction parameters (input variables), ethanol concentration (the mixture of ethanol and distilled water), extraction time, the ratio of ethanol to sample, was evaluated using a central composite RSM model involving six central replicates. Table 2 shows the input variables (extraction para- 
Table 2. Coded values of the variables for the central composite design for cordycepic acid extraction

\begin{tabular}{l|c|c|c|c|c|c}
\hline \multirow{2}{*}{ Variable } & \multirow{2}{*}{ Symbols } & \multicolumn{5}{c}{ Variable levels } \\
\cline { 3 - 7 } & & -1.68 & -1 & 0 & 1 & 1.68 \\
\hline Ethanol concentration [\%] & $X_{1}$ & 26.4 & 40 & 60 & 80 & 93.6 \\
\hline Extraction time [min] & $X_{2}$ & 26.4 & 40 & 60 & 80 & 93.6 \\
\hline Ethanol:sample ratio [ml/g] & $X_{3}$ & 6.6 & 10 & 15 & 20 & 23.4 \\
\hline
\end{tabular}

Table 3. Central composite design matrix for the effect of the variables on the cordycepic acid content in coded values and experimental results

\begin{tabular}{|c|c|c|c|c|c|}
\hline \multirow{2}{*}{ Run } & \multicolumn{3}{|c|}{ Variable } & \multicolumn{2}{|c|}{ Response, Y [mg/g] } \\
\hline & $X_{1}$ & $X_{2}$ & $X_{3}$ & experimental value & predicted value \\
\hline 1 & -1 & -1 & 1 & 31.56 & 29.68 \\
\hline 2 & -1 & 1 & 1 & 33.75 & 31.38 \\
\hline 3 & 1 & -1 & -1 & 19.77 & 19.14 \\
\hline 4 & -1 & -1 & -1 & 8.82 & 6.90 \\
\hline 5 & 0 & 0 & -1.68 & 6.57 & 7.97 \\
\hline 6 & 0 & 1.68 & 0 & 18.16 & 20.55 \\
\hline 7 & 0 & 0 & 1.68 & 25.34 & 28.13 \\
\hline 8 & -1.68 & 0 & 0 & 17.46 & 20.25 \\
\hline 9 & -1 & 1.68 & -1 & 8.25 & 7.64 \\
\hline 10 & 1.68 & 0 & 0 & 17.39 & 19.85 \\
\hline 11 & 0 & -1.68 & 0 & 18.35 & 20.56 \\
\hline 12 & 1.68 & -1 & 1 & 16.95 & 15.33 \\
\hline 13 & 1 & 1 & 1 & 19.95 & 18.66 \\
\hline 14 & 1 & 1 & -1 & 19.08 & 17.44 \\
\hline 15 & 0 & 0 & 0 & 24.91 & 24.68 \\
\hline 16 & 0 & 0 & 0 & 24.95 & 24.68 \\
\hline 17 & 0 & 0 & 0 & 25.48 & 24.68 \\
\hline 18 & 0 & 0 & 0 & 25.02 & 24.68 \\
\hline 19 & 0 & 0 & 0 & 25.15 & 24.68 \\
\hline 20 & 0 & 0 & 0 & 24.78 & 24.68 \\
\hline
\end{tabular}

meters) with their coded and uncoded values. To obtain the optimal extraction conditions, the experiments were carried out according to the experimental design ( Table 3). Table 3 presents the experimental results based on the RSM model. The measured response (cordycepic acid content) was then modeled through an empirical model. A quadratic polynomial equation in terms of the coded variables was established to fit the experimental responses by using the pseudo-inverse technique, as follows:

$$
\begin{aligned}
Y & =24.68-0.12 X_{1}-0.003 X_{2}+6 X_{3}-1.64 X_{1}^{2}-1.46 X_{2}^{2}+ \\
& -2.35 X_{3}^{2}-1.06 X_{1} X_{2}-5.18 X_{1} X_{3}-0.21 X_{2} X_{3}
\end{aligned}
$$

where $X_{3}$ has positive influences on the response; the other parameters have adverse effects.

The central runs (15-20) of repeated experiments showed a low value of the coefficient of variance $(0.98 \%)$, indicating high reproducibility and precision of the experiments. To evaluate the statistical significance of the model, the $\mathrm{F}$ test for ANOVA was examined. As shown 
Table 4. Analysis of variance for the empirical model of cordycepic acid extraction

\begin{tabular}{l|c|c|c|c|c}
\hline \multicolumn{1}{c|}{ Source } & $\mathrm{DF}^{\mathrm{b}}$ & $\mathrm{SS}^{\mathrm{b}}$ & $\mathrm{MS}^{\mathrm{b}}$ & $F$ value & $P>F$ \\
\hline Model $^{\mathrm{a}}$ & 9 & 914.68 & 101.63 & 18.18 & $<0.0001$ \\
\hline Residual (error) & 10 & 55.91 & 5.59 & & \\
\hline Total & 19 & 970.59 & & & \\
\hline
\end{tabular}

${ }^{\mathrm{a}}$ coefficient of determination $\left(R^{2}\right)=0.94$; adjusted $R^{2}=0.89 ;{ }^{\mathrm{b}} \mathrm{DF}-\mathrm{de}-$ gree of freedom; SS - sum of squares; MS - mean square

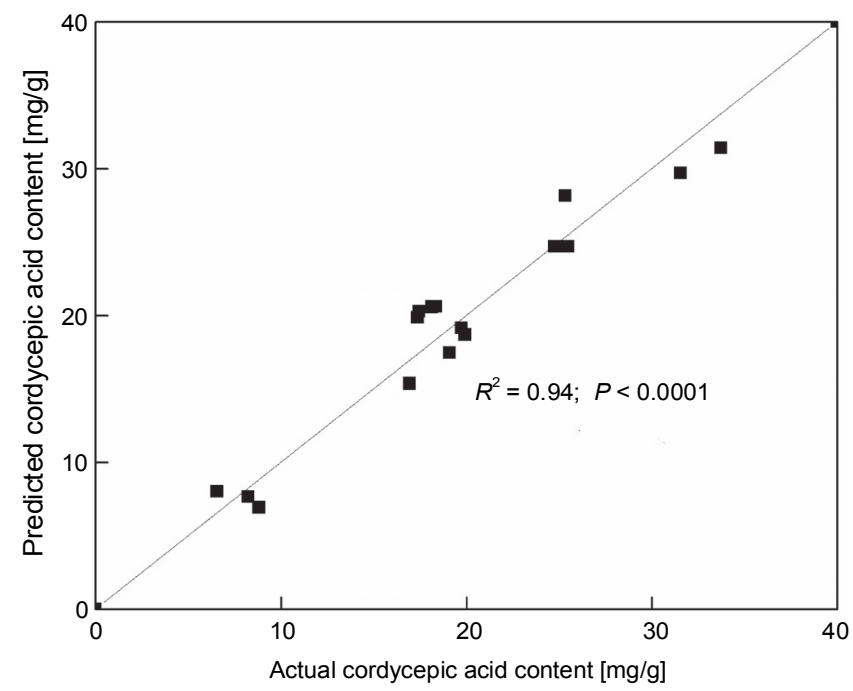

Fig. 2. Correlation between the experimental and predicted cordycepic acid content

Table 5. Significance of the coefficients in the empirical model of cordycepic acid extraction

\begin{tabular}{l|c|c|c|c}
\hline $\begin{array}{c}\text { Model } \\
\text { term }\end{array}$ & $\begin{array}{c}\text { Parameter } \\
\text { estimate }\end{array}$ & $\begin{array}{c}\text { Standard } \\
\text { error }\end{array}$ & $t$ value $^{\mathrm{a}}$ & $P$ value \\
\hline$\beta_{0}$ & 24.68 & 0.96 & 25.77 & $0.000^{\mathrm{b}}$ \\
\hline$\beta_{1}$ & -0.12 & 0.62 & -0.18 & 0.855 \\
\hline$\beta_{2}$ & -0.003 & 0.62 & -0.01 & 0.996 \\
\hline$\beta_{3}$ & 6.00 & 0.65 & 9.23 & $0.000^{\mathrm{b}}$ \\
\hline$\beta_{11}$ & -1.64 & 0.59 & -2.79 & $0.019^{\mathrm{b}}$ \\
\hline$\beta_{22}$ & -1.46 & 0.59 & -2.49 & $0.032^{\mathrm{b}}$ \\
\hline$\beta_{33}$ & -2.35 & 0.62 & -3.78 & $0.004^{\mathrm{b}}$ \\
\hline$\beta_{12}$ & -1.06 & 0.76 & -1.40 & 0.191 \\
\hline$\beta_{13}$ & -5.18 & 0.80 & -6.44 & $0.000^{\mathrm{b}}$ \\
\hline$\beta_{23}$ & -0.21 & 0.80 & -0.26 & 0.804 \\
\hline
\end{tabular}

${ }^{\mathrm{a}} t_{\alpha / 2, n-p}=t_{0.025,10}=2.23 ;{ }^{\mathrm{b}} P<0.05$ indicates that the model terms are significant

in Table 4 , very low $P$ value $(<0.0001)$ of the model in the $\mathrm{F}$ test indicated that the regression is statistically

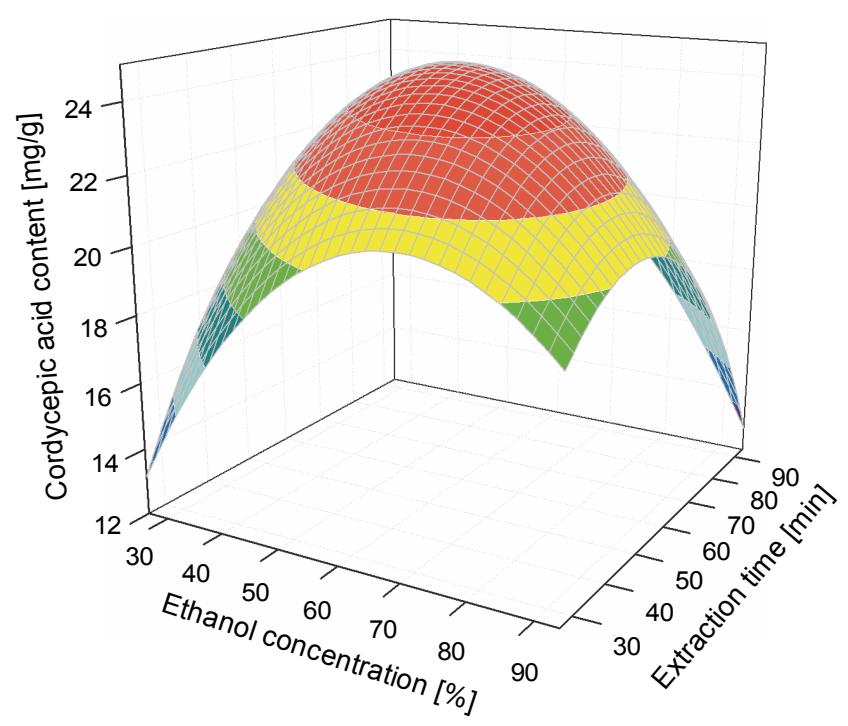

Fig. 3. Response surface plot of the combined effects of the ethanol concentration and the extraction time on the cordycepic acid content at a constant ratio of ethanol to sample $(15 \mathrm{ml} / \mathrm{g})$

significant at the $95 \%$ confidence level. In addition, the quality of the developed model was evaluated using the coefficient of determination $\left(R^{2}\right)$. A high value of $R^{2}$ was obtained (0.94), indicating a high reliability of the model in predicting the cordycepic acid content. Figure 2 and Table 3 show the experimental and predicted values of the cordycepic acid content, which are in strong agreement. This indicated that the established model can provide satisfactory and accurate results for the prediction of the cordycepic acid content. Furthermore, the overall effects of the extraction parameters on the cordycepic acid content were analyzed using $t$ tests (Table 5). Low $P$ values $(<0.05)$ for the intercept term, a linear term $\left(X_{3}\right)$, all quadratic terms, and an interaction term $\left(X_{1} X_{3}\right)$ were obtained, indicating that they significantly affected the extraction efficiency. The developed model was therefore used to plot response surface curves and to predict the optimal extraction conditions for maximizing the cordycepic acid content. 


\section{Effect of extraction parameters on the cordycepic acid content}

Figure 3 illustrates the effects of ethanol concentration and extraction time on the cordycepic acid content while maintaining the ratio of ethanol to sample at its central level $(15 \mathrm{ml} / \mathrm{g})$. The results showed that there was no interaction between the concentration of ethanol and the extraction time. At a given extraction time, the cordycepic acid content increased with an increase in the ethanol concentration. However, a further increase in the concentration of ethanol (> 60\%) resulted in a decrease in the cordycepic acid content (Fig. 3). This is because a high ethanol concentration decreases the polarity of the mixed solvent, thus reducing the solubility of cordycepic acid in the mixed solvent. Studies have demonstrated that the addition of water to ethanol increases the polarity of the ethanol solution (Turkmen et al., 2006; Boulekbache-Makhlouf et al., 2013). Consequently, the mixture of ethanol and water had a higher polarity than absolute ethanol. Therefore, a sufficient ethanol concentration results in the highest solubility of cordycepic acid, thus enhancing the extraction efficiency. In this study, low ethanol concentrations $(<60 \%)$ were found to be efficient for the extraction of cordycepic acid from the fruiting body of $C$. militaris.

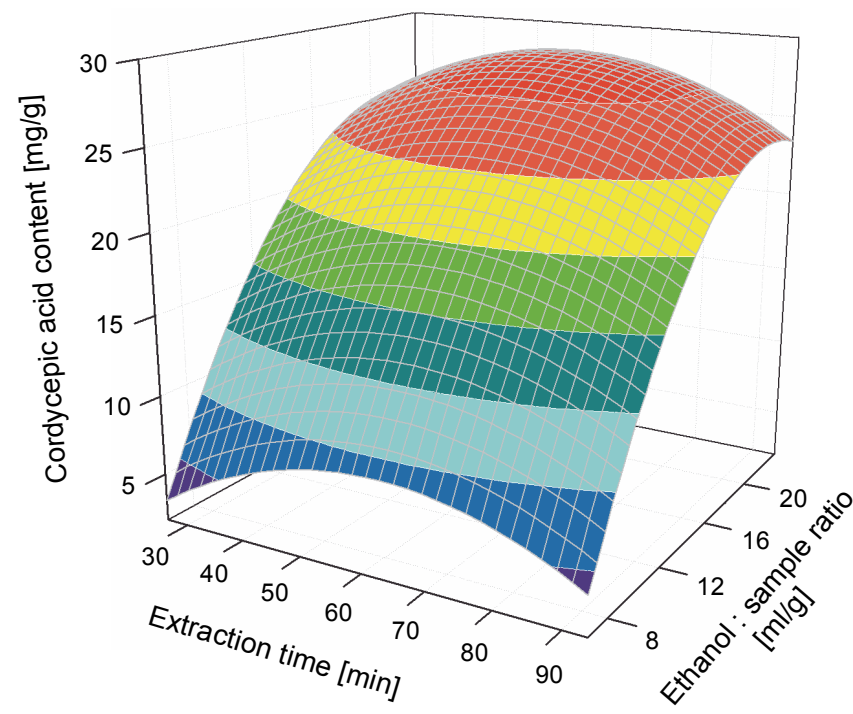

Fig. 4. Response surface plot of the combined effects of the extraction time and the ratio of ethanol to sample on the cordycepic acid content at a constant ethanol concentration (60\%)

Figure 4 presents the effects of the extraction time and the ratio of ethanol solution to the sample on the cordycepic acid content. At any extraction time, the cordycepic acid content significantly increased when the ratio of ethanol to sample increased. This could be because the extraction of cordycepic acid from the cell is easy at a high liquid: solid ratio. Studies have demonstrated that a high amount of solvent can provide a sufficient environment for the extraction of metabolite compounds from the biomass (Kostić et al., 2013; Wang et al., 2013). In addition, the solubility of cordycepic acid can increase at a high ratio of ethanol to sample, thus enhancing the extraction efficiency (Xiao et al., 2009). Furthermore, the extraction time significantly affected the extraction of cordycepic acid (Fig. 4). The cordycepic acid content was observed to reach the highest value $(38.68 \mathrm{mg} / \mathrm{g})$ at an extraction time of $69.8 \mathrm{~min}$ and then decrease with further increase in the extraction time. This reduction could be due to the degradation of cordycepic acid at longer extraction times.

Table 6. The maximal cordycepic acid content under optimal extraction conditions

\begin{tabular}{l|c}
\hline & $\begin{array}{c}\text { Cordycepic acid content } \\
{[\mathrm{mg} / \mathrm{g}]}\end{array}$ \\
\hline Predicted value using RSM & 38.68 \\
\hline Experimental validation & $40.23 \pm 0.16^{\mathrm{a}}$ \\
\hline
\end{tabular}

${ }^{a}$ Data are expressed as the mean $\pm \mathrm{SD}$ of three replications

\section{Obtaining optimal extraction conditions}

The root of the empirical model (Eq. 2) for the cordycepic acid content represents the maximization of the response function. The canonical method was employed to predict the optimal extraction conditions for maximizing the cordycepic acid content. The maximal cordycepic acid content was predicted to be $38.68 \mathrm{mg} / \mathrm{g}$ at an ethanol concentration of $26.4 \%$, an extraction time of $69.8 \mathrm{~min}$, and a ratio of ethanol to sample of $23.4: 1 \mathrm{ml} / \mathrm{g}$ (Table 6). An experiment was then conducted under the optimal extraction conditions to verify the predictions. A cordycepic acid content of $40.23 \pm 0.16 \mathrm{mg} / \mathrm{g}$ was obtained (Table 6), indicating agreement with the empirical model prediction. Thus, the developed RSM model can be used to adequately represent the relationship between the variables and the response in the extraction of cordycepic acid from the fruiting body of $C$. militaris. In addition, the cordycepic acid content in this study was 
higher than that in the previous study $(24.7 \mathrm{mg} / \mathrm{g}) \mathrm{re}-$ ported by Deng et al. (2013), in which cordycepic acid was extracted from $C$. militaris using a microwave-assisted extraction process. The result of our research suggested that the extraction method obtained in this study is a suitable process for extraction of cordycepic acid from the fruiting body of $C$. militaris.

\section{Conclusions}

This study reports the extraction of cordycepic acid from the fruiting body of $C$. militaris by using different solvents. Of the solvents tested, ethanol was identified as the most effective solvent for the extraction of cordycepic acid. The extraction conditions were subsequently optimized for maximizing the cordycepic acid content using RSM. The optimal extraction conditions were determined using RSM, and a cordycepic content of $40.23 \mathrm{mg} / \mathrm{g}$ was obtained. The results suggest that the method obtained in this study is promising for the extraction of cordycepic acid from the fruiting body of C. militaris.

\section{References}

Bao F., Tang R., Cheng L., Zhang C., Qiu C., Yuan T., Zhu L., Li H., Chen L. (2018) Terpenoids from Vitex trifolia and their anti-inflammatory activities. J. Nat. Med. 72: 570-575.

Boulekbache-Makhlouf L., Medouni L., Medouni-Adrar S., Arkoub L., Madani K. (2013) Effect of solvents extraction on phenolic content and antioxidant activity of the byproduct of eggplant. Ind. Crops Prod. 49: 668-674.

Das S.K., Masuda M., Sakurai A., Sakakibara M. (2010) Medicinal uses of the mushroom Cordyceps militaris: current state and prospects. Fitoterapia 81: 961-968.

Deng L., Zhou T.Y., Pi L., Zhao X.H., Han T., Li Y.K., Han F. (2013) Optimization of microwaveassisted extraction of cordycepic acid and cordycepin from cultured cordyceps militaris by response surface methodology. Asian J. Chem. 25: 8065-8071.

Do Q.D., Angkawijaya A.E., Tran-Nguyen P.L., Huynh L.H., Soetaredjo F.E., Ismadji S., Ju Y.H. (2014) Effect of extraction solvent on total phenol content, total flavonoid content, and antioxidant activity of Limnophila aromatica. J. Food Drug Anal. 22: 296-302.

Franco R.R., Justino A., Silva H., Peixoto L., Espindola F. (2018) Antioxidant and anti-glycation capacities of some medicinal plants and their potential inhibitory against digestive enzymes related to type 2 diabetes mellitus. J. Ethnopharmacol. 215: 140-146.

Jin Y., Meng X., Qiu Z., Su Y., Yu P., Qu P. (2018) Anti-tumor and anti-metastatic roles of cordycepin, one bioactive compoundofCordyceps militaris. Saudi J. Biol. Sci. 25: 991-995.
Kostić M.D., Joković N.M., Stamenković O.S., Rajković K.M., Milić P.S., Veljković V.B. (2013) Optimization of hempseed oil extraction by n-hexane. Ind. Crops Prod. 48: 133-143.

Kuppusamy P., Yusoff M.M., Parine N.R., Govindan N. (2015) Evaluation of in-vitro antioxidant and antibacterial properties of Commelina nudiflora L. extracts prepared by different polar solvents. Saudi J. Biol. Sci. 22: 293-301.

Lin S., Liu Z.Q., Xue Y.P., Baker P.J., Wu H., Xu F., Teng Y., Brathwaite M.E., Zheng Y.G. (2016) Biosynthetic pathway analysis for improving the cordycepin and cordycepic acid production in Hirsutella sinensis. Appl. Biochem. Biotechnol. 179: 633-649.

Nguyen H.C., Liang S.H., Doan T.T., Su C.H., Yang P.C. (2017) Lipase-catalyzed synthesis of biodiesel from black soldier fly (Hermetica illucens): optimization by using response surface methodology. Energy Conver. Manage. 145: 335-342.

Shih L., Tsai K.L., Hsieh C. (2007) Effects of culture conditions on the mycelial growth and bioactive metabolite production in submerged culture of Cordyceps militaris. Biochem. Eng. J. 33: 193-201.

Soltani M., Malek R.A., Ware I., Ramli S., Elsayed E., Aziz R., El-Enshasy H. (2017) Optimization of cordycepin extraction from Cordyceps militaris fermentation broth. J. Sci. Ind. Res. 76: 355-361.

Song J.F., Li D.J., Liu C.Q. (2009) Response surface analysis of microwave assisted extraction of polysaccharides from cultured Cordyceps militaris. J. Chem. Technol. Biotechnol. 84: 1669-1673.

Tahmouzi S., Ghodsi M. (2014) Optimum extraction of polysaccharides from motherwort leaf and its antioxidant and antimicrobial activities. Carbohydr. Polym. 112: 396-403.

Tang J., Qian Z., Wu H. (2018) Enhancing cordycepin production in liquid static cultivation of Cordyceps militaris by adding vegetable oils as the secondary carbon source. Bioresour. Technol. 268: 60-67.

Turkmen N., Sari F., Velioglu Y.S. (2006) Effects of extraction solvents on concentration and antioxidant activity of black and black mate tea polyphenols determined by ferrous tartrate and Folin-Ciocalteu methods. Food Chem. 99: 835-841.

Wang H.J., Pan M.C., Chang C.K., Chang S.W., Hsieh C.W. (2014) Optimization of ultrasonic-assisted extraction of cordycepin from Cordyceps militaris using orthogonal $\mathrm{ex}$ perimental design. Molecules 19: 20808-20820.

Wang L., Huang Q.H., Huang Y.F., Xie J.H., Qu C., Chen J.P., Zheng L., Yi T.G., Zeng, H.F., Li, H.L. (2018a) Comparison of protective effect of ordinary Cordyceps militaris and selenium-enriched Cordyceps militaris on triptolide-induced acute hepatotoxicity and the potential mechanisms. J. Funct. Foods 46: 365-377.

Wang S., Dong X., Tong J. (2013) Optimization of enzymeassisted extraction of polysaccharides from alfalfa and its antioxidant activity. Int. J. Biol. Macromol. 62: 387-396.

Wang X., Huang H., Ma X., Wang L., Liu C., Hou B., Yang S., Zhang L., Du G. (2018b) Anti-inflammatory effects and 
mechanism of the total flavonoids from Artemisia scoparia Waldst. et kit. in vitro and in vivo. Biomed. Pharmacother. 104: 390-403.

Xiao J.H., Xiao D.M., Xiong Q., Liang Z.Q., Zhong J.J. (2009) Optimum extraction and high-throughput detection of cordycepic acid from medicinal macrofungi Cordyceps jiangxiensis, Cordyceps taii and Cordyceps gunnii. J. Food Agric. Environ. 7: 328-333.
Złotek U., Mikulska S., Nagajek M., Świeca M. (2016) The effect of different solvents and number of extraction steps on the polyphenol content and antioxidant capacity of basil leaves (Ocimum basilicum L.) extracts. Saudi J. Biol. Sci. 23: $628-633$ 